\title{
Characteristics Recognition and Soft Multimedia System for Japanese Machine Translation and Edge-Driven Hardware Implementations
}

\section{Gang Song ( $\sim$ wajj18964@163.com )}

Beijing Foreign Studies University

\section{Research Article}

Keywords: Japanese Teaching, Machine Translation, Feature Recognition, Neural Network, Statistical Translation, Multimedia System, Hardware Structure

Posted Date: May 27th, 2021

DOl: https://doi.org/10.21203/rs.3.rs-524468/v1

License: (c) (i) This work is licensed under a Creative Commons Attribution 4.0 International License. Read Full License 


\section{Abstract}

With the development of economic globalization, international exchanges and cooperation are increasingly frequent and in-depth. In this process, there is a serious obstacle, that is, language differences. Therefore, the development of high quality and practical machine translation system is of great significance. Japan has a close relationship with China, so it is necessary to acquire and process Japanese information. Japanese translation is the basis of Japanese information processing, which plays an important role in cross language information retrieval, machine translation, information extraction and other practical applications. In recent years, machine translation has made remarkable progress, but there is still much room for improvement in the quality of translation. Multimedia assisted instruction is an important application of computer technology in teaching field. In recent years, the wide application of multimedia courseware in all kinds of teaching has received good teaching results. With the development and deepening of Japanese teaching reform, contextualized teaching has been paid more and more attention. In this context, Japanese intensive teaching is also increasingly showing the trend of network, multimedia assisted. Therefore, this paper realizes the feature recognition and hardware structure of Japanese machine translation.

\section{Introduction}

With the rapid increase of information in today's world, the communication and cooperation between people who use different languages are more and more frequent and in-depth. There is a serious obstacle to such communication and cooperation, that is, language differences. With the rapid popularization of computer network, people can get information in various fields in time through the network, but the information on the network is carried by various languages. Therefore, in order to overcome the language barrier and realize information sharing, machine translation is an important way to solve the problem. Automatic translation of different languages is not only a tool of communication, but also an important means of cultural exchange and communication. Therefore, the study of machine translation has important practical significance and application value for information sharing and information exchange [1-4].

With the development and deepening of Japanese teaching reform, contextualized teaching has been paid more and more attention. In teaching. The CAI system can display the materials conveniently, create the best situation and get the best teaching effect. At present, Japanese has become one of the most important foreign languages after English in China. With the development of information technology in education, Japanese education also needs to be diversified to meet the needs of the times. As one of the required courses for college students majoring in foreign languages, Japanese translation is an important course integrating theory and practice. In the information age, traditional teaching methods can not meet the needs of modern teaching, so multimedia assisted teaching has become the future development trend of teaching activities. 
Machine translation (MT) is the application of computers to realize the translation from one natural language text to another. Machine translation system (MTS) is a tool that human use computer to translate and process natural language automatically. Machine translation system is a multi-disciplinary and complex computer software system. Its implementation involves linguistics, mathematics and computer science, covering the three knowledge fields of Arts, science and engineering [5-8].

Machine translation is a process of transforming two languages by using computer software on the basis of maintaining semantic consistency. Machine translation belongs to the subfield of computational linguistics, and the combination of artificial intelligence and natural language processing is its important research content. Since the emergence of machine translation task in 1940s, machine translation has experienced two stages: early rule-based machine translation and recent statistical machine translation. Since neural network research was used in machine translation in 2014, the use of end-to-end neural machine translation has developed rapidly. This technology adopts a new method system, and directly uses neural network to map the source language text to the target language text. Different from statistical machine translation, neural machine translation does not need word alignment, phrase segmentation and other steps, nor linguistic knowledge support such as syntactic analysis. Neural machine translation has the advantages of low labor cost and short development cycle, and it overcomes the problems of semantic representation and error propagation in statistical machine translation.

The traditional teaching method of translation course, which takes teachers as the center and book content as the main line, can not meet the needs of the new form. It is imperative to study and explore a new teaching mode that takes learners as the center and computer courseware as the assistant of translation course. For many years, there has always been a single teaching problem in translation teaching, among which the lack of class hours is the main reason for the singleness of translation teaching. The lack of teaching time makes it impossible for teachers to conduct translation teaching in a deeper and wider way. In the classroom teaching, teachers rely only on the existing teaching materials and supplement a lot of information collected on the Internet, which is not only very difficult, but also requires a lot of teaching time. Using multimedia courseware can show the supplementary content to students in the form of courseware, which not only saves time, but also enriches the classroom content. Based on the correct selection of materials and the development of courseware, the multimedia courseware should be used in the teaching of translation course reasonably, so as to achieve the expected teaching effect.

In order to improve the quality of machine translation, automatic error detection and classification plays an important role in MT output post-processing. On the one hand, it can help post editors improve their work efficiency, on the other hand, it can analyze the translation difficulty of the corresponding source language according to translation errors. The source language input is transformed to decode again, and then the translation performance is improved. Therefore, translation error judgment, classification and analysis is one of the important research contents of SMT technology development and application. At present, most translation error detection methods use system features such as word posterior probability (WPP) to estimate translation confidence, and use shallow syntactic or semantic knowledge as 
assistance to reduce classification error rate and improve error prediction performance. In terms of system features, many WPP methods based on N-best or word graph calculation for confidence estimation are proposed, and have been widely used in machine translation error detection. After that, some researchers try to use other knowledge sources as features and combine them with WPP confidence features to detect errors, such as deep syntactic or deep semantic features. However, due to the complexity of external features and the relevance of specific language in most cases, effective and universal features are not easy to be extracted. Therefore, at present, lexical features such as posteriori probability, word and part of speech still play a major role [9-12].

\section{A Study Of Japanese Machine Translation 2.1. The Development and Current Situation of Machine Translation}

The study of machine translation began in the late 1940s. In the course of more than 50 years' development, MT research has experienced several stages, such as initial stage, depression stage, recovery stage and prosperity stage.

In 1946, booth, a British engineer, and Weaver, the vice president of Rockefeller Foundation in the United States, put forward the idea of automatic language translation when discussing the application scope of computers. In this period, it is believed that the study of machine translation should be done more in syntactic analysis. The feature of machine translation is to separate grammar from algorithm, which is also a great progress of machine translation technology. Machine translation in the recovery period is divided into six steps: lexical analysis, syntactic analysis, lexical conversion, syntactic conversion, syntactic generation and translation generation.

China is one of the first countries engaged in machine translation research in the world. In 1957, the Institute of language and computation of the Chinese Academy of Sciences took the lead in the research of Russian Chinese machine translation. Since then, many scholars have invested in this research field, and have carried out the research of French Chinese, German Chinese, Japanese Chinese and other machine translation, and made some achievements.

Direct translation is achieved through word translation, insertion, deletion and local word order adjustment, without deep syntactic and semantic analysis. Syntactic transformation and semantic transformation are similar. Their translation process can be divided into three stages: analysis, transformation and generation. In the analysis stage, the source language sentence is analyzed and the deep structure of the sentence is analyzed. For syntactic transformation, the deep structure is mainly syntactic information. For semantic transformation, the deep structure is mainly semantic information. In the transformation stage, the deep structure of the source language is transformed into the deep structure of the target language. Finally, the target language sentence is generated from the deep structure of the target language. The interlanguage method uses an inter-language as the intermediary representation of 
translation, and divides the whole translation process into two stages: analysis and generation. In the analysis phase, convert the source language to the intermediate language. In the generation phase, the intermediate language is converted to the target language. Among them, the analysis process is only related to the source language, not the target language. The generation process is only related to the target language, not the source language.

In the early machine translation system, the methods used can be divided into direct translation, conversion and intermediate language. The difference of these methods lies in the depth of the source language analysis. The same thing is that they all need a lot of rules, such as language conversion rules, source language derivation rules, target language generation rules and so on, as well as large-scale bilingual dictionaries. Among them, the transformational method is the most profound in the analysis of source language, which includes morphological analysis, structural analysis and semantic analysis, and completes the transformation of structure, semantic and morphological three-tier structure from source language to target language. The transformational method takes into account the characteristics of the source language and the target language, and it is easier to get good translation results than the intermediate language method. Therefore, the early translation system adopted the translation method of transformation, and the whole translation process was divided into three parts: source language generation, transformation and generation.

\subsection{Machine Translation Methods}

The knowledge representation of rule-based machine translation is rule. Its advantages are: the rule granularity has great scalability; the rule with large granularity has strong generalization ability, and the rule with small granularity has very fine description ability. Rule-based machine translation can express linguists' knowledge directly. This method has strong adaptability, does not depend on specific training corpus, and can integrate different linguistic features. A rule-based translation method uses a set of rules to understand natural language. This set of rules includes three aspects: the source language analysis rules that describe the source language, the conversion rules from the source language to the target language, and the generation rules that generate the target language.

Therefore, the core of rule-based translation is to construct a complete or adaptable rule system. But such a complete set of rules system is difficult to construct. Because of the poor coverage of the rules, especially the rules of fine particle size are difficult to summarize comprehensively. The subjective factors of the rules are strong, sometimes there is a certain gap with the objective facts. With the increasing number of rules, there is no good solution to the conflict between rules. In the end, rules are highly dependent on the language and only apply to specific systems, so the development and maintenance cost of rule base is too high.

Rule-based translation method has achieved good results in some specific restricted areas, but in most experiments, it has not met the requirements of people. With the rapid development of corpus linguistics and the widespread application of statistics and information theory in the field of natural language processing, researchers began to try to use statistical methods for machine translation. 
The statistical based translation method is derived from the translation idea of "decoding ciphers", which belongs to the noise channel model. The source channel model is also called noise channel model. The kernel and idea of noise channel model is to regard the process of machine translation as a process of information transmission of a piece of text. The process of decoding the information using the noise channel model is shown in Fig. 1.

After passing the noise channel, the target text $t$ becomes the source language $S$. the decoding process is to restore the source language $s$ to the target language $t$. The method based on noise channel model uses language model and translation model to represent translation probability. $\mathrm{P}(\mathrm{T})$ is the probability of the occurrence of target text $\mathrm{T}, \mathrm{P}(\mathrm{S} \mid \mathrm{T})$ is the probability of $\mathrm{t}$ being translated into $\mathrm{s}$, the former is the language model, the latter is the translation model. The language model reflects the characteristics of the target language. It refers to the probability that the text sequence t may appear in the target language, that is, whether it conforms to the syntax rules of the target language. The translation model reflects the relationship between bilingualism and refers to the probability that the target language $t$ is the source language s translation.

According to Bayesian formula, the above decoding process can be deduced as follows:

$$
T=\underset{T}{\arg \max } P(T) P(S \mid T)
$$

The advantage of statistical based machine translation is that it is not dependent on language. As long as there is a corpus, it is easy to adapt to new fields or languages. The system has short development cycle and good robustness. The system does not need to write rules manually, and uses corpus to train the machine translation system directly. Although the translation quality of statistical method is better, it has some disadvantages. First of all, it costs a lot of time and space. Secondly, data sparsity is a serious problem. Finally, it is difficult to integrate different linguistic features and introduce complex linguistic knowledge. Therefore, how to construct large-scale aligned bilingual corpus and how to estimate the parameters of the model are the key problems to be solved. In addition, designing a search algorithm with good performance is helpful to find the best translation.

In the case-based machine translation system, the bilingual translation case base is the main knowledge source of the system. There are two fields in the translation case base, one is to save the sentences in the source language, and the other is to save the corresponding translation. When entering a sentence of a source language, the system compares the sentence with the sentence field of the source language in the instance database. The system finds out the most similar sentence with this sentence, simulates the translation corresponding to this sentence, and finally outputs the translation.

In the case-based machine translation system, translation knowledge is represented in the form of examples and bilingual dictionaries, which is easy to add or delete, and the maintenance of the system is also easy. If a large translation case base is used and a precise comparison is made, it is possible to produce a high-quality translation, and the difficulty of deep linguistic analysis in traditional rule-based 
machine translation methods is avoided. However, the case-based method also has disadvantages, that is, low coverage. A practical system needs a large corpus. For case-based machine translation, there are two aspects to be studied. One is bilingual automatic alignment. In the case base, the corresponding target language instances and instance fragments should be accurately found from the source language instances and instance fragments. In the specific implementation of the case-based machine translation system, not only the alignment at the sentence level, but also the alignment at the vocabulary level or even the phrase or sentence structure level is required. The other is the case matching retrieval. Because of the large scale of the case base, in order to quickly find the case or instance segment matching the sentence to be translated in the case base, an efficient retrieval mechanism needs to be established. In addition, the matching of instance and instance fragment is usually not exact matching, but fuzzy matching. Therefore, to determine whether two sentence or phrase fragments are similar, we need to establish a set of similarity criteria.

Since the development of case-based translation, there are two more special methods: translation memory method and template based method. The process of translation memory can be described in this way. First, save the translated sentences. Then, when translating new sentences, we can directly find them in the corpus. If we find the same sentences, we can directly output the translation; otherwise, we will give them to someone to translate, but we can provide a reference translation of similar sentences. Such a method is usually used in computer-aided translation software. Template based translation is a method between rules and instances. In terms of knowledge representation, it is more concrete than rule knowledge and more abstract than instance knowledge. Templates are rules of lexicalization.

Monolingual templates are strings of constants and variables, while translation templates are composed of two corresponding monolingual templates and variable mapping relationship between them.

Phrase based translation is a hot topic in the field of statistical machine translation. This method is better than the word based statistical machine translation method in performance, because it can better grasp the local context dependency.

Phrase based translation is developed from word based translation. This model is based on the corpus of word alignment. It regards any continuous word string as a phrase, and then learns the bilingual phrase pair according to the bilingual corpus of word alignment, and counts the translation probability of the phrase. This method translates according to the probability table of phrase translation. For a given sentence in the source language, the translation process is as follows: first, the sentence in the source language is divided into phrases; second, each phrase is translated according to the translation model; finally, the translated phrases are reordered.

\subsection{Decoding Algorithm}

In statistical machine translation, there are different decoding algorithms according to different translation models. At present, the most commonly used search algorithm is column search, and the decoding process mainly includes four steps: 
(1) Given a source language string, all possible translation results are obtained through phrase translation table. In the target language, the translations corresponding to these phrases are found, and translation candidates and corresponding translation probabilities are generated.

(2) According to the translation candidates generated in the first step, the translation probability is calculated to minimize the cost of the untranslated part.

(3) Column search is used to search for translation candidates, and pruning strategy is used to optimize the process. Finally, the search results are traced back and the target language translation $t$ is generated.

Pruning strategies include merging pruning and histogram pruning. Merging pruning is a pruning strategy that saves the cost of two search paths in the process of merging, and its data will not lose information. However, due to the exponential change of the number of sentences in the search space, it is impossible

to get the global optimal solution in the shortest possible time. Therefore, the pruning strategy of heuristic search is introduced. Histogram pruning is a risky pruning strategy. In the process of pruning, the inappropriate hypothesis is deleted and the search space is greatly reduced, which reduces the time complexity of decoding algorithm.

\subsection{Feature Extraction in Japanese Machine Translation}

In the decoding part of the hierarchical phrase model, CKY algorithm is used to reduce the source language sentence to the start symbol and find out the best derivation to get the target language sentence. The hierarchical phrase model combines a variety of features, and uses the minimum error rate training to train the feature weight.

Hierarchical phrase model is one of the best statistical translation models at present. Its main advantages include the following points. Firstly, the hierarchical phrase model integrates lexicalization features and contextual information to guide translation. Secondly, the hierarchical phrase is a kind of formal syntactic structure, so it does not need linguistic knowledge and the method of hierarchical rule extraction is simple. Hierarchical phrases can be easily applied between any two languages. Finally, the model has a certain generalization ability, which to some extent alleviates the problem of long-distance order adjustment.

Through the syntactic analysis, we can get the syntactic analysis tree which reflects the lexical dependency. How to use the information of these syntactic trees effectively has always been a hot issue in machine translation. There are two kinds of translation models based on linguistic syntax: one is based on phrase syntax tree, the other is based on dependency tree. There are also three types of syntactic tree information used in Translation: tree to string model, string to tree model and tree to tree model.

Phrase component tree is a kind of generative grammar, which refers to the phrase blocks that can form a certain meaning between consecutive word sequences. Dependency tree refers to the dependency relationship between words whose predicate verb is core and other constituent words. The phrase 
component tree contains more syntactic information, while the tree structure of dependency tree is simple and clear. Compared with the component tree, the number of rules in the dependency tree is reduced. Figures 2 and 3 are examples of phrase component trees and dependency syntax trees, respectively.

The biggest advantage of the method based on syntax tree is the introduction of syntactic structure knowledge. Compared with the formal syntactic model which can only be used for local phrase ordering, the model based on syntactic tree has the ability of global phrase ordering, which is in line with people's intuitive expression of language understanding. But at the same time, the main disadvantage of this kind of model is that it depends on syntactic analysis, so the accuracy of syntactic analysis directly affects the accuracy of translation model. Secondly, due to the different syntactic tree structures, the translation rules in the syntactic model are very large and the decoding is time-consuming, so it cannot be applied in the application scenarios with high real-time requirements for translation.

\section{Multimedia System Construction And Hardware Implementation Of Machine Translation}

\subsection{Multimedia Classroom Publishing Teaching}

In the information age, traditional teaching methods can not meet the needs of modern teaching, multimedia assisted teaching has become the future development trend of teaching activities. Task driven teaching was first seen in German "dual system" teaching, which aims to cultivate students' mental skills or operational skills. In the middle and late 20th century, with the continuous development of education and information technology, task driven teaching has become an important teaching trend. The Federal Institute of Vocational Education in Germany has developed a task driven teaching method which is "student-centered". The traditional teaching mode will be changed into multi-dimensional interactive teaching mode, so as to promote students' active exploration, learning practice, self reflection, and improve students' thinking ability, innovation ability and the ability to cooperate with others. In the United States, the task-driven approach has been adopted since the beginning of primary education. Teachers combine the teaching content to design specific small tasks, which require students to complete in groups. Students can complete tasks through various channels. The final task results are displayed in the class, and teachers and students comment and exchange together. Faridah Musa (2011) introduced task-driven teaching method into translation class, and gradually taught students the necessary skills for the workplace. Paul Jackson (2008), Kuoshu Huang (2012) and other scholars at home and abroad have also tried to use task-driven teaching as a necessary supplement to traditional classroom teaching. Using information technology to serve the classroom teaching and make the teaching courseware play a great role, which is the trend of modern teaching development. The capacity of this kind of computer teaching content is incomparable with that of traditional teaching.

From speech recognition to statistical machine translation confidence estimation, word posterior probability has always been a major and effective feature of confidence estimation. In terms of statistical machine translation, given the source language input, the posterior probability of a word refers to the 
probability that the word will occur in the target language sentence, that is, the output of machine translation. In a general sense, the basic idea is to give the source language input. If the probability (or frequency) of a word in the translation hypothesis of the target language is very high, it is very likely to be a correct translation. But the actual situation shows that the calculation based on the posterior probability of words in mathematical sense can not really reflect whether the target words are correct or not. The factors that affect the accuracy of posterior probability of target words are as follows:

(1) The coverage of training corpus or translation model to source language input. Too low coverage will lead to a large number of extraneous words appearing in translation hypothesis, which makes the posterior probability of the extraneous words calculated according to the N-best list higher.

(2) The domain adaptability of training corpus and source language input. The difference in data domain will result in the great difference in meaning expression between translation hypothesis and source language input. The probability of target words with inaccurate meaning expression appearing in N-best list may be very high, which leads to low intelligibility of translation hypothesis.

\subsection{Hardware Initialization of Machine Translation System}

The system initialization process can be divided into three main parts: chip level initialization, board level initialization and system level initialization.

Chip level initialization completes the initialization of embedded microprocessor, including setting the core register and control register of embedded microprocessor, the core working mode of embedded microprocessor and the local bus mode of embedded microprocessor. Chip level initialization gradually sets the embedded microprocessor from the default state when it is powered on to the required working state of the system. This is a hardware only initialization process.

Board level initialization completes the initialization of hardware devices other than embedded microprocessors. In addition, some software data structures and parameters need to be set up to establish hardware and software environment for subsequent system level initialization and application program operation. This is an initialization process including both hardware and software.

System initialization the initialization process is mainly software initialization, mainly for the initialization of the operating system. BSP transfers the control of embedded microprocessor to the embedded operating system, and the operating system completes the remaining initialization operations. Initialization includes loading device drivers, establishing system memory area, loading and initializing other system software modules, such as network system, file system, etc. Finally, the operating system creates the application environment and gives control to the application's portal.

Another main function of BSP is hardware related device driver. Initialization of hardware related device drivers is usually a process from high to low. Although BSP contains hardware related device drivers, these device drivers are usually not directly used by BSP, but in the process of system initialization, BSP 
associates them with the general device drivers in the operating system, and in subsequent applications, they are called by the general device drivers to realize the operation of hardware devices.

GUI provides users with a graphical interface and manages many windows and layers. At the same time, the GUI receives the underlying messages sent by the system, such as the coordinates of the mouse and the key values of the keys. Judge according to these elements, and then readjust the display content to achieve the role of handover with users.

At present, the common GUI on the embedded platform are MiniGUI, QT / embedded, nano-x windows system, GTK, open GUI, PicoGUI, ucGUI, etc. Among them, MiniGUI and QT are widely used.

The software level of MiniGUI is shown in the figure below.

The application program based on MiniGUI generally realizes its own functions through ANSI C library, operating system and driver interface and API provided by MiniGUI itself. The portable layer in MiniGUI hides the details of specific operating system and underlying hardware, while the upper application does not need to care about the underlying hardware platform output and input devices.

\subsection{Hardware Scheme Design}

Telechips is a chip R \& D company in South Korea. The chip scheme is mainly used in MP3, PDA, mobile phone and other multimedia terminal equipment, with remarkable characteristics in audio and video processing. We choose the chip of tccc8300 produced by it for development. The chip has the following characteristics:

ARM926EJ-S core, high $220 \mathrm{MHz}$ processing speed.

Provide external SDRAM, SRAM, nor flash, prom interface, and internal integration of 64KB SRAM.

The internal logic structure of the chip is shown in Fig. 5.

The main functions are as follows:

1. Realize intelligent translation between multiple languages. Due to the large size of the library files needed for statistical machine translation, NAND flash with large capacity must be selected as the external memory.

2. Text browsing. In the text browsing or word searching interface, any sentence can be selected for intelligent reading, so the speech synthesis chip SC-691 is needed.

3. USB charging is supported, and ISL6292 is selected for power management.

4. It supports standby mode and requires two external frequency crystal oscillators: high frequency crystal oscillator for normal working state and low frequency crystal oscillator for standby. 
5. Realize the decoding of audio, video and picture.

WM8987 has a built-in high-quality DAC, which is used to convert digital audio signals into audio signals that can push headphones or speakers to output. In addition, WM8987 also has digital noise filtering, EQ audio equalizer, volume control, electronic switch, 3D sound effect, hardware high and low tone control and other processing units.

The input of WM8987 includes two sets of analog input (input1, input2) and one set of digital input. Digital input adopts the situation of IIS bus. Two groups of analog inputs are divided into left and right channels. At the same time, after the configuration of internal registers, the sampling of a single channel can be realized. Therefore, it can support up to 4 mono analog inputs.

Using the machine translation system based on the statistical method, it is easy to achieve the translation between multiple languages, so only the corresponding corpus can be added. But corpora need a large storage space, usually the size of a corpus is hundreds of megabytes, so NAND flash of $2 \mathrm{G}$ size is selected.

The circuit schematic diagram of NAND flash module is shown in Fig. 7.

\section{Experiment And Analysis \\ 4.1. Experimental Data Analysis}

Experimental design:

(1) This paper classifies three typical posterior probability features of words, compares their performance and analyzes them.

(2) The large entropy model is used to classify individual linguistic features.

(3) Three typical posterior probability features of words are combined with linguistic features respectively to carry out classification experiments, and to compare and analyze them.

The classification experiment results based on the posterior probability of words are shown in Table 1 . 
Table 1

Results of three typical WPP features for translation error detection

\begin{tabular}{|lllll|}
\hline Features & CER/\% & $\mathrm{P} / \%$ & $\mathrm{R} / \%$ & $\mathrm{~F} / \%$ \\
\hline Baseline & 41.61 & & & \\
\hline WPP_Dir & 40.68 & 63.49 & 72.45 & 67.66 \\
\hline WPP_Win & 39.71 & 63.81 & 73.96 & 68.50 \\
\hline WPP_Lev & 40.12 & 60.22 & 92.08 & 72.88 \\
\hline
\end{tabular}

Among them, WPP_Dir represents the posteriori probability feature of words based on fixed position; WPP_Win represents the posteriori probability feature of words based on sliding window; WPP_Lev represents the posteriori probability feature of words based on leven shtein alignment.

The error detection results based on linguistic features, POS and link are shown in Table 2.

Table 2

Performance of the error detection task based on linguistic features

\begin{tabular}{|lllll|}
\hline Linguistic features & CER/\% & $\mathrm{P} / \%$ & $\mathrm{R} / \%$ & $\mathrm{~F} / \%$ \\
\hline Baseline & 41.51 & & & \\
\hline Word & 39.12 & 64.22 & 76.68 & 69.08 \\
\hline POS & 39.52 & 61.53 & 86.47 & 71.29 \\
\hline Link & 40.88 & 59.92 & 93.55 & 72.77 \\
\hline
\end{tabular}

In terms of $\mathrm{F}$ value, Link is superior to the other two features, and POS is superior to Word.

(1) The classification ability of combined features is better than that of individual features, and the linguistic features and source word features can improve the statistical MT translation error detection ability to a certain extent.

(2) After adding the source word feature, its F value also decreased to some extent.

(3) The combination of WPP based on word alignment and other four features gets the best results, which shows the importance of location information and rich word alignment information for error detection and classification.

In this experimental system, users can input English sentences for Japanese-English translation. The results are shown in Fig. 8.

\section{Conclusion}


As far as the current level of development is concerned, the translation quality of machine translation is far from reaching and replacing the level of human translation. Therefore, there is still much room for theoretical research and system implementation of machine translation, and further research on machine translation is of great theoretical value and practical significance. At present, neural machine translation has achieved great success, and its effect in many language pairs has surpassed statistical machine translation. Since 2014, MT has produced a large number of scientific research results and practical products. Due to the short time of research, there are still many problems worthy of further exploration. Compared with statistical machine translation, the results of neural machine translation have a greater improvement in sentence fluency. At the same time, the fusion of linguistic knowledge is essential for the improvement of neural machine translation performance. In the new era, Japanese teaching reform is urgent. The focus of Japanese teaching has gradually shifted from teachers' classroom teaching to students' ability of independent thinking. This paper constructs a multimedia system and hardware implementation of Japanese machine translation. The application of multimedia classroom publishing teaching method is not only the embodiment of Japanese teaching reform, but also the application of the combination of science and technology and education.

\section{Declarations}

\section{Conflict of Interest}

The authors declare no conflicts of interest regarding to publish this paper.

\section{Ethics approval and consent to participate}

Not applicable.

\section{Consent for publication}

Not applicable.

\section{Competing interests}

The authors declare that they have no competing interests

\section{Acknowledgement}

The research was supported by the Beijing Social Science Fund Project (No. 19YYB011).

\section{References}


1. Chen Zhangming. Some thoughts on making multimedia courseware to assist teaching [J]. China Vocational and technical education, 2001.

2. Li Kedong, Xie Youru. Multimedia instructional design [EB / OL]. Instructional design course learning network.

3. He Kekang et al. Teaching system design [M]. Beijing Normal University Press, 2002.

4. Zhang Li. The whole of streaming media technology [M]. China Youth Press, 2001.

5. Li Yunlin, Xu Fuyin. Theory and practice of teaching media [M]. Beijing Normal University Press, 2004.

6. Duff $M$, Chen $Y$, Attygalle $S$, et al. An adaptive mixed reality training system for stroke rehabilitation[J]. Neural Systems and Rehabilitation Engineering, IEEE Transactions on, 2010, 18(5): 531-541.

7. Wu Peiwu, Xu Jinan, Xie Jun, et al. Chunk based dependency tree to cross Japanese Chinese statistical machine translation model [J]. Acta Sinica Sinica, 2014, 28 (5): 133-140.

8. Chen Bi. Research on machine translation technology based on neural network [D]. University of Chinese Academy of Sciences, 2016.

9. Feng Zhiwei. Machine translation; from rule-based technology to statistics based technology. Proceedings of 2010 China translation professional exchange conference, 2010.

10. Li Ronglu, Wang Jianhui, Chen Xiaoyun, et al. Classification of Chinese texts using the maximum fu organ model [J]. Computer research and development, 2005, 42 (1): 94-101.

11. Zhou Jun, He Li, Han Weihong, et al. An Empirical Study on the classification of Chinese comment propensity based on machine learning. proceedings of the 28th National Conference on computer security 2013: 164-166.

12. He Shengyong. On the application of multimedia classroom publishing in Japanese teaching and countermeasures [D]. Chongqing University, 2017.

13. Li Xinchen. The combination of language and culture in Japanese Teaching [J]. Literature education (2), 2018 (09): 30-31.

14. Zheng Jie. An analysis of Japanese Teaching Innovation Based on online learning mode [J]. Drama House, 2017 (24): 189.

15. Xu Xiaoxu. On the situational application of euphemism as a pragmatic strategy in Japanese Teaching [J]. Journal of Jilin radio and Television University, 2018 (09): 151-152.

16. Wang Xuanli. The joint application of multimedia and College Japanese Teaching [J]. Literature education, 2013.

\section{Figures}




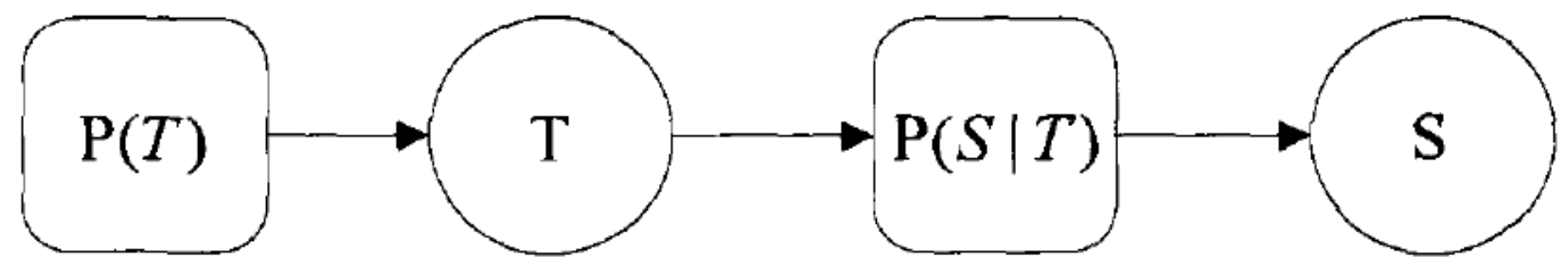

Figure 1

Noise channel model

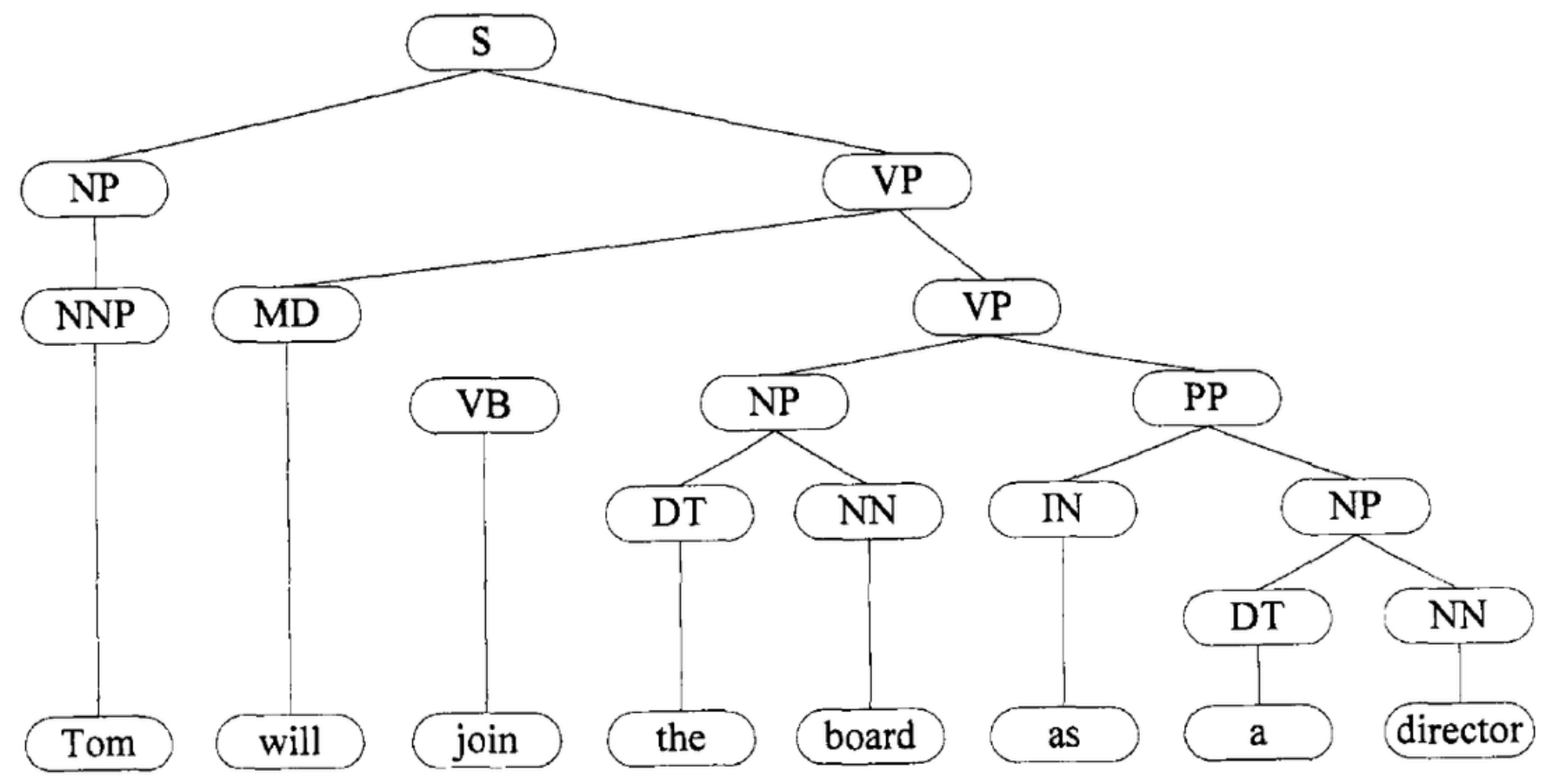

Figure 2

An example of syntax tree 


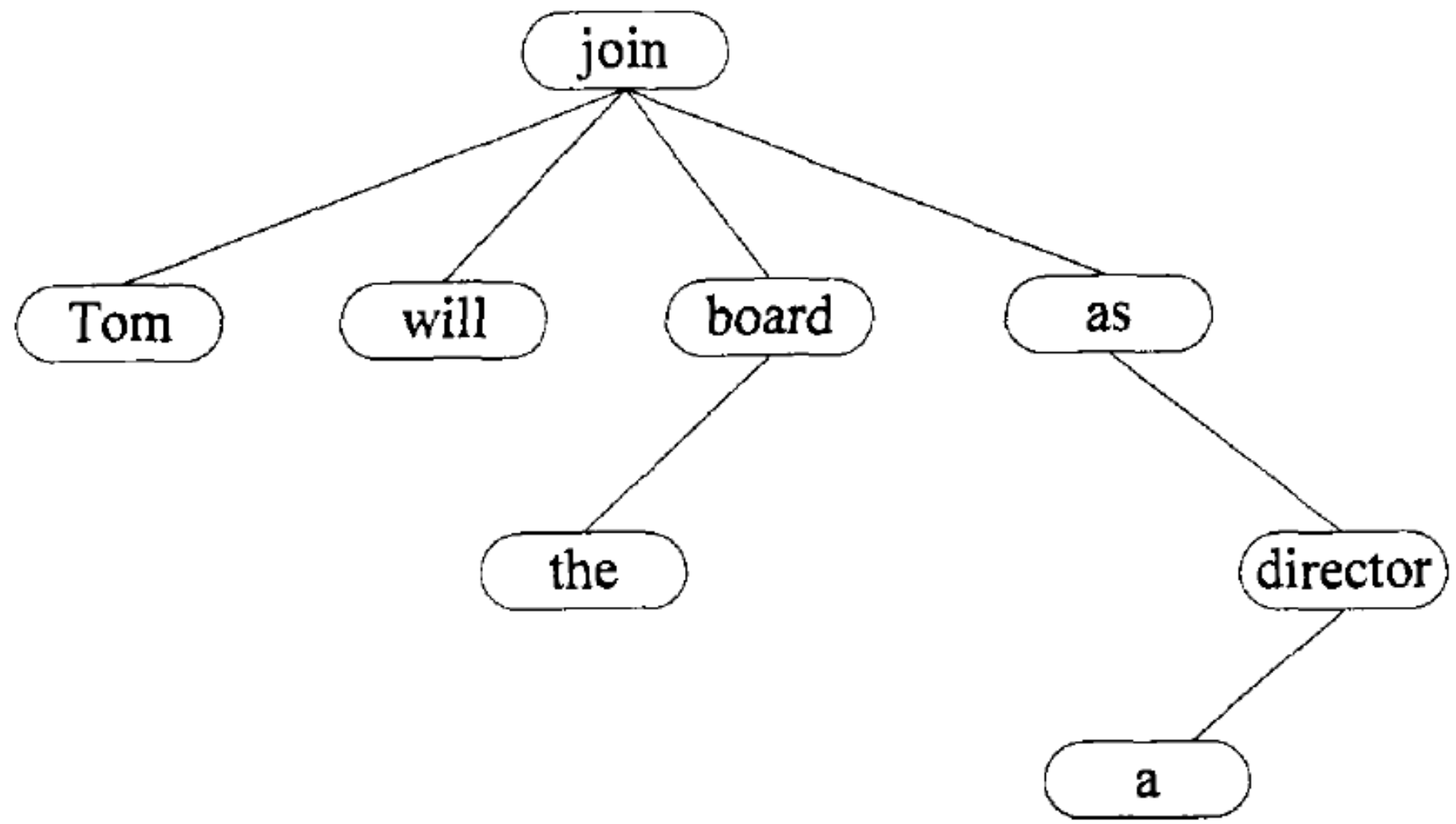

Figure 3

An example of dependency tree 


\section{MiniGUI Applications}

\section{MiniGUI Core}

Portable Layer

ANSI C

Library

Devices

Linux/uClinux,VxWorks, ThreadX,eCos,uCos-II... ix86,ARM,MIPS,PowerPC,M68K...

Figure 4

Software level of MiniGUI 


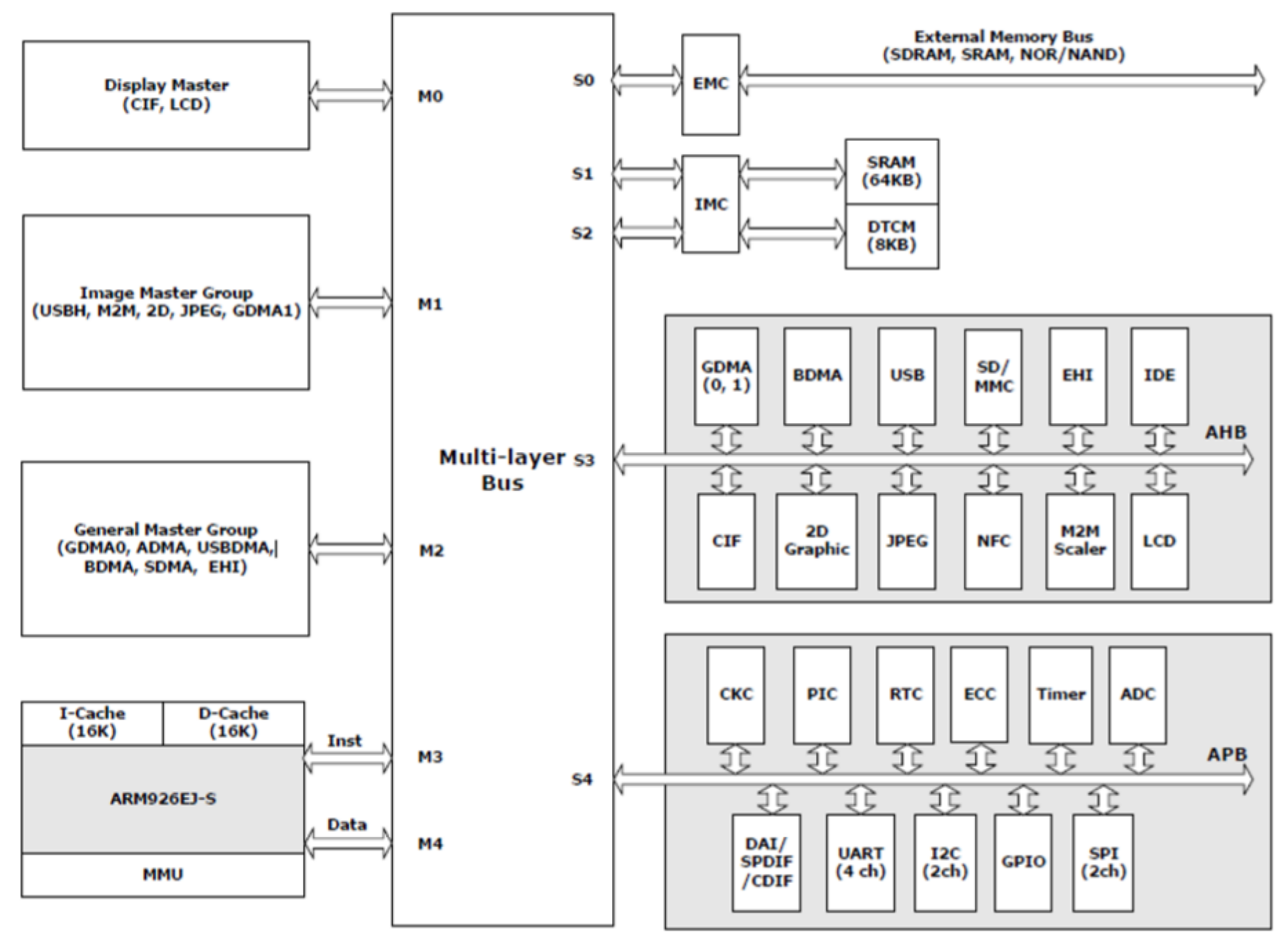

Figure 5

Internal logic structure of chip 


\begin{tabular}{|c|c|c|c|c|}
\hline $\begin{array}{l}\text { REGISTER } \\
\text { ADDRESS }\end{array}$ & BIT & LABEL & DEFAULT & DESCRIPTION \\
\hline $\begin{array}{c}\mathrm{R} 31(1 \mathrm{FH}) \\
\mathrm{ADC} \text { input mode }\end{array}$ & $7: 6$ & $\begin{array}{c}\text { MONOMIX } \\
{[1: 0]}\end{array}$ & 00 & $\begin{array}{l}00: \text { Stereo } \\
01 \text { :using left ADC } \\
\text { 10:using right ADC } \\
11: \text { Digital Mono Mix } \\
00: \text { Normal mode }\end{array}$ \\
\hline $\begin{array}{c}\mathrm{R} 23(17 \mathrm{H}) \\
\text { Additional control }\end{array}$ & $3: 2$ & $\begin{array}{c}\text { DATSET } \\
{[1: 0]}\end{array}$ & 00 & $\begin{array}{l}01: \text { left \& right data }=\text { left } \mathrm{ADC} \\
10: \text { left \& right data }=\text { right } \mathrm{ADC} \\
11: \text { swap left right data }\end{array}$ \\
\hline $\begin{array}{c}\mathrm{R} 32(20 \mathrm{H}) \\
\text { ADC signal path } \\
\text { control (Left) }\end{array}$ & $7: 6$ & LINSEL & 00 & $\begin{array}{l}00: \text { LINPUT1 } \\
01: \text { LINPUT2 } \\
10: \text { Reserved } \\
11: \text { L-R differential }\end{array}$ \\
\hline
\end{tabular}

\section{Figure 6}

Partial register description of WM8987

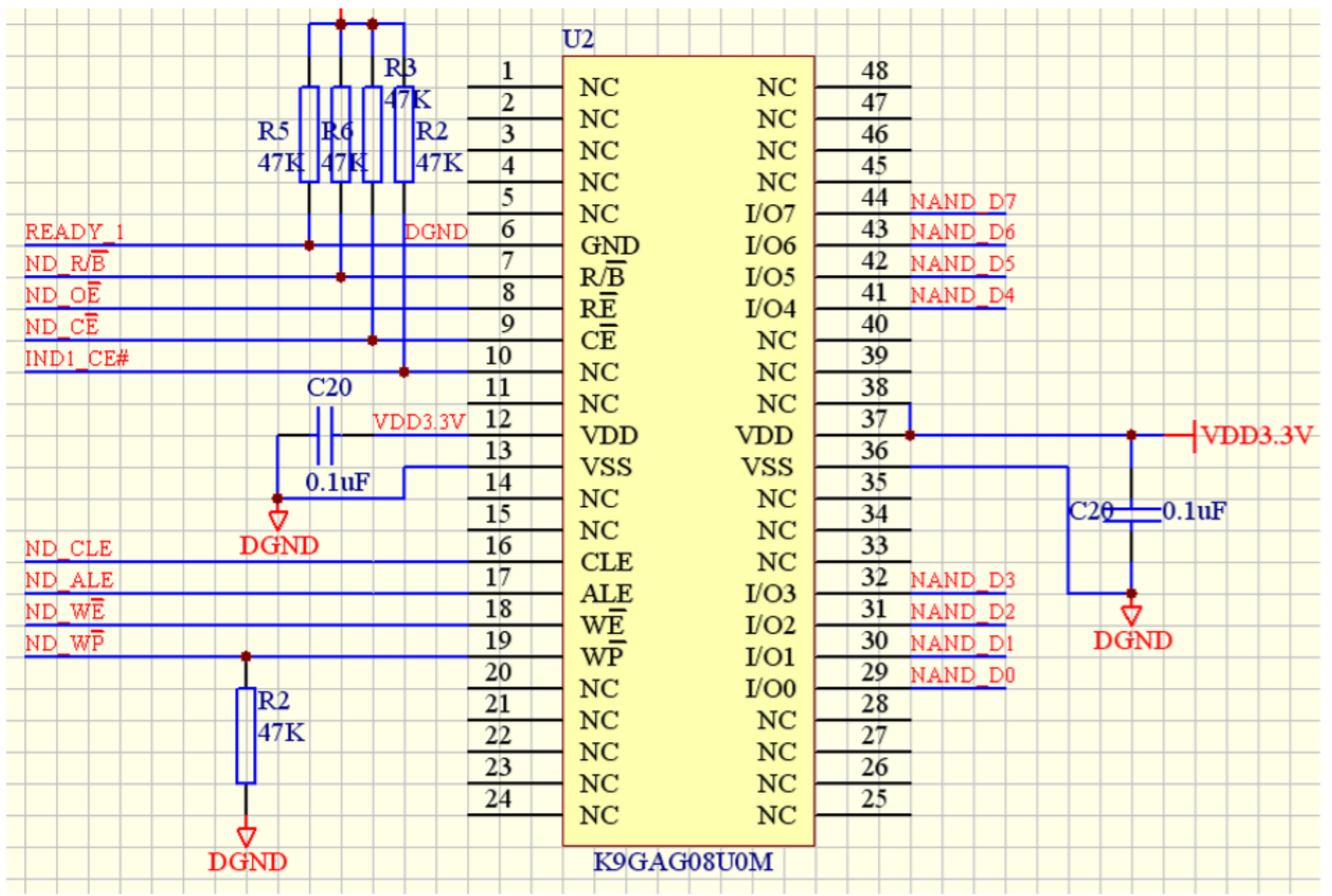

Figure 7 
NAND flash module circuit diagram

good morning

would you repeat that

when the boys won the race I was very glad

of course, everyone began to shout very loudly

somethimes people ask me about my name

everyone is going into class

what about your family

\section{Figure 8}

\section{Example of translation}

グッドモーニング

あなたは繰り返す

少年がレースに勝ったとき、私はとてもうれしかった

もちろん、みんな大声で叫び始めた

人々は私の名前について私に尋ねる

誰もがクラスに入る

ご家族について 